\title{
High resolution STM imaging with oriented single crystalline tips
}

\author{
A. N. Chaika ${ }^{a}, *$, S. S. Nazin ${ }^{a}$, V. N. Semenov ${ }^{a}$, N. N Orlova ${ }^{a}$, S. I. Bozhko ${ }^{a, b}$, O. Lübben ${ }^{b}$, \\ S. A. Krasnikov ${ }^{\mathrm{b}}$, K. Radican ${ }^{\mathrm{b}}$, and I. V. Shvets ${ }^{\mathrm{b}}$ \\ ${ }^{a}$ Institute of Solid State Physics RAS, Chernogolovka, Moscow district 142432, Russian Federation \\ ${ }^{b}$ Centre for Research on Adaptive Nanostructures and Nanodevices, School of Physics, Trinity College, Dublin 2, Ireland
}

\begin{abstract}
Precise knowledge of the atomic and electronic structure of scanning tunneling microscopy (STM) tips is crucial for correct interpretation of atomically resolved STM data and improvement of spatial resolution. Here we demonstrate that tungsten probes with controllable electronic structure can be fabricated using oriented single crystalline tips. High quality of the [001]-oriented W tips sharpened in ultra high vacuum was proved by electron microscopy. Distance dependent STM studies carried out on graphite (0001) surface demonstrate that application of crystallographically oriented single crystalline tips allows one to control the tip electron orbitals responsible for high resolution imaging under specific tunneling conditions.
\end{abstract}

PACS: 68.37.Ef; 73.40.Gk; 73.20.At

Keywords: STM imaging, Electron orbital, Tungsten, Graphite

\section{Introduction}

High spatial resolution of scanning tunneling microscopy (STM) [1,2] is based on the tunneling current exponential dependence on the distance between the sample and the probe tip. This allows direct visualization of the surface electronic structure with atomic resolution [3]. The exponential current-distance dependence means that the tunneling current in STM experiments is mainly collected by the front tip atom. Therefore, detailed knowledge of its electronic structure is particularly important. In most studies, unknown probe structure is modeled by a spherically symmetric $s$-wave tip [4]. However, this simplification may not be valid in general case because different electron orbitals can contribute to the tunneling current. According to the theory of C. J. Chen [5], individual tunneling matrix elements are proportional to derivatives of the corresponding wave functions. Sums of these individual orbital contributions can be responsible for usual spherically symmetric atomic features but asymmetry effects can be observed in experiments if electron orbitals with non-zero orbital momentum $(l)$ and momentum projection $(\mathrm{m})$ on the quantization axis dominate at the tip apex. It took approximately two decades after the invention of STM to perform direct measurements of the asymmetric charge distribution related to apex atomic orbitals in atomic force microscopy (AFM) [6,7] and STM [8-12] experiments. Visualization of the $f_{z}^{3}$ orbital $(l=3, m=0)$ associated with $\mathrm{Sm}$ atom at the apex was claimed in Ref. [8]; however, one could speculate whether the observed halos and extremely sharp surface atomic features reproduce the shape of the tip electron 
orbital. Recently, two legs of the MnNi tip $d_{x z}$ orbital $(l=2, m=1)$ were resolved in STM experiments on the $\mathrm{Cu}(014)-\mathrm{O}$ surface but only in a narrow range of bias voltages and tunneling currents $[10,11]$. The surface complexity did not allow to reveal the conditions where the $d_{x z}$ orbital channels most of the tunneling current: Distance dependent contribution of the surface electron states with different $m$ led to essential modification of atomically resolved STM images and chemically selective visualization of copper and oxygen rows of the $\mathrm{Cu}(014)-\mathrm{O}$ surface $[11,13]$.

In this work, we use a highly oriented pyrolytic graphite (HOPG) surface and [001]-oriented single crystalline $\mathrm{W}$ tips with predictable atomic and electronic structure to study relative contribution of different electron orbitals of the apex atom at different bias voltages and tip-surface separations. The results of STM experiments and density functional theory (DFT) calculations demonstrate that relative contribution of different electron orbitals can be controlled in precise distance dependent experiments with single crystalline tips. This is confirmed by reproducible imaging of extremely sharp spherically symmetric and four-fold split subatomic features at different tunneling parameters.

\section{Experimental details}

STM experiments were carried out in constant current mode using an ultra high vacuum (UHV) room temperature microscope GPI-300. The [001]-oriented W tips were fabricated from $0.5 \times 0.5 \times 10 \mathrm{~mm}^{3}$ single crystalline bars using electrochemical etching in $2 \mathrm{M} \mathrm{NaOH}$ solution. Although $\mathrm{W}(001)$ is a natural cleavage plane in the tungsten crystal, most of the chemically etched tips possessed sharp apexes with typical radii of curvature in the range of $5-30 \mathrm{~nm}$. To produce clean W[001] probes suitable for controllable high resolution STM experiments we used the tip preparation procedure [14] consisting of consecutive electron beam flash heating and $\mathrm{Ar}^{+}$ion sputtering in the UHV STM chamber (base pressure $p<1 \times 10^{-10}$ mbar). According to ex-situ high resolution transmission electron microscopy (TEM) data (Fig. 1), this UHV treatment allowed us to produce sharp [001]-oriented tungsten tips with nanoscale pyramids at the apex. TEM image shown in Fig. 1(b) reveals $\{001\}$ and $\{011\}$ crystallographic planes at the [001]oriented tungsten apex. In most cases, atomic resolution could be achieved on HOPG(0001) and more complex surface reconstructions (e.g., see Ref. [14] and Figs. 2 and 3) with in-situ cleaned W[001] tips without additional treatments which could modify their atomic structure. Tunneling current dependences of the HOPG(0001) STM images were measured at several fixed sample bias voltages to study relative contributions of different tip electron orbitals at different tip-sample distances. Using the reciprocity principle of STM [15], in this case we could probe the orbital structure of the W[001] tip atom using more spatially localized carbon $s$ and $p_{z}$ orbitals of the surface atoms. All distance dependent studies were carried out only with clean $\mathrm{W}[001]$ tips suitable for atomic resolution experiments immediately after the tip to sample approach. Tips were refreshed in case of occasional mass transport from the sample to the tip apex. 


\section{Results and discussion}

The occupied surface state STM image of the $\operatorname{Si}(111) 7 \times 7$ reconstruction shown in Fig. 2(a) provides a typical example of high resolution imaging with insitu cleaned single crystalline W[001] tips (Fig. 1). The cross-section in Fig. 2(b) illustrates that rest atoms, lying in the second layer of the $7 \times 7$ reconstruction [Fig. 2(c)], are well resolved as individual protrusions located at approximately the same height as the top layer adatoms. According to theoretical calculations [16], the rest atoms can only be resolved in STM experiments with extremely sharp single atom terminated tips at certain bias voltages.

Figure 3 shows two typical atomically resolved STM images of the

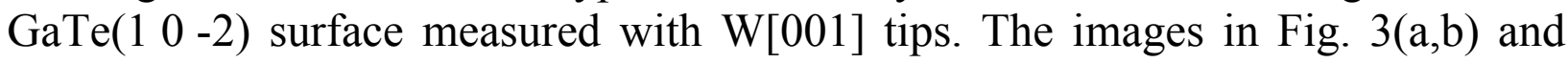
$3(\mathrm{c}, \mathrm{d})$ reveal chemically selective visualization of the electronic features associated with either $\mathrm{Te}$ or $\mathrm{Ga}$ atoms in the topmost layer. Additional experimental and theoretical studies are necessary to identify the nature of the observed chemical selectivity achieved at fixed sample bias voltage. Although possible modifications of the tip state could not be excluded, different spatial distribution of $\mathrm{Ga}$ and $\mathrm{Te}$ atomic orbitals and/or some modification of the electronic structure of the surface atoms interacting with the tip atom can be the reason of the chemically selective imaging. Distance dependent STM experiments with single crystalline W[001] tips on the graphite (0001) surface reveal possible changes in the electronic structure of the interacting tip and surface atoms at small distances. In this case the effect is most ??? pronounced for the tip electron states. Note that sharp single crystalline tip with controllable apex geometry (Fig. 1) yielding atomic resolution on complex surfaces (Fig. 2,3) is crucial to probe the orbital structure of the frontmost tip atom.

According to the calculated partial density of electron states (PDOS) associated with the apex atom of the isolated W[001] tip (e.g., see [5,13] and references therein), $d_{3 z-r}^{2}$ and $d_{x-y}^{2}{ }^{2}$ states dominate in the density of occupied and empty electron states near $\mathrm{E}_{\mathrm{F}}$, respectively. In this case, small positive sample bias voltages would be optimal for achieving highest lateral resolution because of large PDOS associated with the $d_{3 z-r}^{2}{ }^{2}$ tip states slightly below $\mathrm{E}_{\mathrm{F}}$. In contrast, at small negative voltages one can expect a significant contribution from nonzero $m\left(d_{x-y}^{2}{ }^{2}\right)$ electron states. These two situations for the tip orbital selection are schematically shown in Fig. 4.

In our STM experiments on $\mathrm{HOPG}(0001)$ we could reproducibly resolve [17] extremely sharp spherically symmetric features at small positive sample bias voltages [Fig. 5(b)] and four-fold split subatomic features at small negative voltages [Fig. 5(a)] which could be explained by decisive contribution of the tip electron states with different $m$. However, selection of the tip orbital is controlled by both the bias voltage and the gap resistance as demonstrated by the tunneling current dependences taken at two fixed bias voltages (Fig. 5). Both sharp symmetric and split subatomic features could be resolved only at high tunneling currents (small gap resistances) corresponding to small tip-sample separations and strong interaction in the W-graphite system.

To explain the distance dependence of the HOPG(0001) STM images measured with W[001] tips, we performed the ab-initio DFT calculations of the 
interacting tungsten-graphite system using the VASP program. A plane augmented wave basis [18] was used with the energy cutoff of $400 \mathrm{eV}$. The electron exchange and correlation was simulated by local density approximation pseudopotentials with the Ceperley-Alder exchange functional [19]. A $\Gamma$ centered $(1 \times 1 \times 1) \mathrm{k}$-point grid was used to sample the Brilliouin zone. The global break condition for the electronic self consistent loops was set to a total energy change of less than $1 \cdot 10^{-6}$ $\mathrm{eV}$. To model the $\mathrm{W}$ tip we used a [001]-oriented pyramid of seven unit layers (128 atoms in total) with the apex atom positioned above the surface $\beta$-atom which does not have the nearest neighbor in the second layer. The simulated graphite slab consisted of six unit layers. The initial atomic positions in the tip and surface clusters were chosen in accordance with the bulk lattice parameters of tungsten and graphite crystals. DFT calculations were carried out for several gaps between interacting tip and surface atoms in the range of 1.5--5.0 $\AA$. The distance between the back of the tip cluster and the graphite slab for these tip-surface separations was between $25 \AA$ and $21.5 \AA$ that eliminated interaction between the back sides of the tip and surface clusters. Atomic positions for the interacting tip-surface system were optimized by relaxing six layers of the W[001] tip and five layers of the graphite surface. Only atomic positions in the back layers of the tip and surface clusters were fixed during the calculations. Electron density of states (DOS) was calculated for several pre-optimized tip-surface structures. As an example, Fig. 6a shows the results of PDOS calculations for three tip-sample separations of 4.92, 2.58, and $2.39 \AA$. These distances between the interacting tip and surface atoms correspond to initial separations of $4.0 \AA, 2.25 \AA$, and $1.75 \AA$ in non-relaxed system and vacuum gaps of $22.5 \AA, 24.25 \AA$, and $24.75 \AA$ between non-interacting back sides of the tip and surface clusters.

The PDOS calculations reveal that the tip $d_{3 z-r}^{2}$ electron orbital possesses the largest DOS near $E_{F}$ at tip-surface distances between $2.5 \AA$ and $4.0 \AA$ [e.g., the PDOS at $2.58 \AA$ separation is shown in Fig. 6(a)]. This is in line with the gap resistance dependence measured at a bias voltage of $23 \mathrm{mV}$ [Fig. 5(b)] demonstrating substantial enhancement of the lateral resolution with increasing current (decreasing distance). Note that apparent diameters of extremely sharp spherically symmetric atomic features resolved at large tunneling currents [right panel of Fig. 5(b)] correlate with the known spatial distribution of carbon electron states proving imaging carbon atomic orbitals by the tungsten $d_{3 z-r}{ }^{2}{ }^{2}$ electron orbital. The PDOS associated with the $d_{3 z-r}{ }_{-2}^{2}$ orbital is substantially suppressed at small distances when overlapping of the tungsten and carbon atomic orbitals takes place. As the top panel in Fig. 6(a) illustrates, at distances below $2.5 \AA$ the $d_{x y}$ orbital of the $\mathrm{W}$ tip atom could provide the largest contribution to the tunneling current at small negative sample bias voltages producing the asymmetric four-fold split subatomic features [right panel of Fig. 5(a)] reproducibly observed in our experiments at high tunneling currents. Note that substantially simpler DFT calculations for constrained W[001]-graphite system [17] produced qualitatively the same results predicting the suppression of the $d_{3 z-r}{ }^{2}$ orbital at distances below $2.5 \AA$. 
Calculated charge density maps shown in Fig. 6(b,c) demonstrate that the STM data [Fig. 6(d,e)] can be explained by direct visualization of the W[001] tip atom electronic structure modified at small tunneling gaps by the tip-sample interaction. DFT calculations for the coupled tip-sample system reveal asymmetric charge density maps around the $\mathrm{W}[001]$ tip atom at very small distances from the apex atom core [Fig. 6(c)] and symmetric charge distribution at slightly larger distances [Fig. 6(b)] which correlate with the experimental features [Fig. 6(d,e)]. The tunneling parameters applied in the STM experiment suggest that a minor $(0.2-0.3 \AA)$ decrease in the tip-sample distance could be responsible for the observed transformation from spherically symmetric [Fig. 6(d)] to four-fold split [Fig. 6(e)] subatomic features. This is in agreement with the results of PDOS calculations [Fig. 6(a)] showing the suppression of $d_{3 z-r}^{2}{ }^{2}$ orbital at the W[001] tip atom with the decrease in the tunneling gap from $2.58 \AA$ to $2.39 \AA$. Although the exact values of tip-sample separations can hardly be obtained from the tunneling parameters, the gap resistance dependence of STM images (Fig. 5) and results of the DFT calculations [Fig. 6(a)] allow us to suggest that tunneling gaps in the range of 2.5-2.8 $\AA$ and 2.2-2.5 $\AA$ could be responsible for the images shown in Figs. $6(d)$ and $6(e)$, respectively.

\section{Conclusions}

We have demonstrated that relative contribution of different tip electron orbitals in STM experiments can be controlled by employing stable single crystalline probes with predictable atomic and electronic structure. The experimental data obtained with [001]-oriented $\mathrm{W}$ tips and results of DFT calculations prove that selective imaging of particular atomic orbitals can be achieved by carefully adjusting the bias voltage and tip-sample distance. We have shown that imaging at the level of individual electron orbitals can be done even at room temperature and details of intra-atomic electronic structure can be revealed reproducibly in careful distance dependent experiments. The effect has been demonstrated for the tip orbitals, but the situation can be reversed when imaging the sample surface electronic structure with the STM probe featuring $s$ - or $p_{z}$-type orbitals at the tip apex atom.

\section{Acknowledgements}

This work was supported by the Russian Academy of Sciences, Russian Foundation for Basic Research (grant 11-02-01256), SFI E.T.S. Walton Programme, and Marie Curie IIF grant within the 7th European Community Framework Programme. A.Ch. thanks L.V. Yashina, A. Volykhov and S. Vassiliev for providing GaTe samples and help in experiments.

\section{References}

[1] G. Binnig et al., Appl. Phys. Lett. 40 (1982) 178.

[2] G. Binnig and H. Rohrer, Helv. Phys. Acta 55 (1982) 726.

[3] G. Binnig et al., Phys. Rev.Lett. 49 (1982) 57. 
[4] J. Tersoff and D.R. Hamann, Phys. Rev. Lett. 50 (1983) 1998; Phys. Rev. B 31 (1985) 805.

[5] C.J. Chen, Phys. Rev. Lett. 65 (1990) 448; Phys. Rev. B 42 (1990) 8841; Phys.

Rev. Lett. 69 (1992) 1656.

[6] F.J. Giessibl et al., Science 289 (2000) 422.

[7] S. Hembacher et al., Science 305 (2004) 380.

[8] M. Herz et al., Phys. Rev. B 68 (2003) 045301.

[9] L.A. Zotti et al., Chem. Phys. Lett. 420 (2006) 177.

[10] A.N.Chaika et al., Phys. Rev. Lett. 98 (2007) 206101.

[11] S. Murphy et al., Phys. Rev. B 76 (2007) 245423.

[12] A.N. Chaika and A.N. Myagkov, Chem. Phys. Lett. 453 (2008) 217.

[13] A.N. Chaika, S.S. Nazin and S.I. Bozhko, Surf. Sci. 602 (2008) 2078.

[14] A.N. Chaika, V.N. Semenov, S.S. Nazin and S.I. Bozhko, Appl. Phys. Lett. 95 (2009) 173107.

[15] C. J. Chen, Introduction to Scanning Tunneling Microscopy, 1993, Oxford University Press, New York.

[16] Y.L. Wang et al., Phys. Rev. B 70 (2004) 073312.

[17] A.N. Chaika et al., EPL 92 (2010) 46003.

[18] G. Kresse and J. Furthmüller, Phys. Rev. B 54 (1996) 11169.

[19] D.M. Ceperley and B.J. Alder, Phys. Rev. Lett. 45 (1980) 566.

[20] I. Horcas et al., Rev. Sci. Instrum. 78 (2007) 013705. 
Figures and captions.

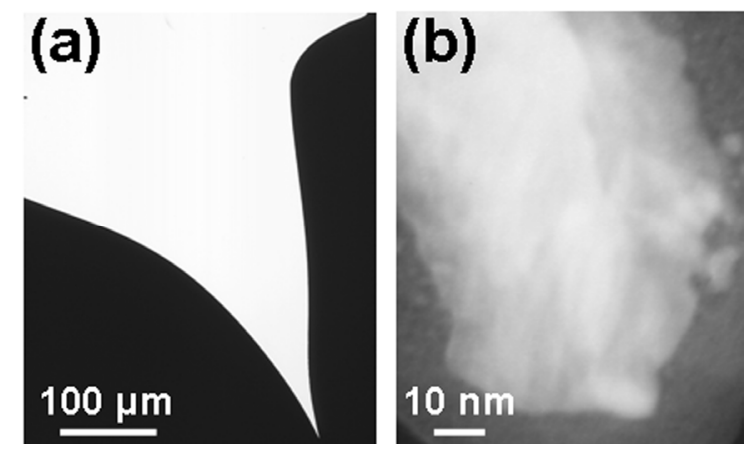

Fig. 1. (a,b) TEM images of a W[001] tip after electron beam heating and co-axial ion sputtering.

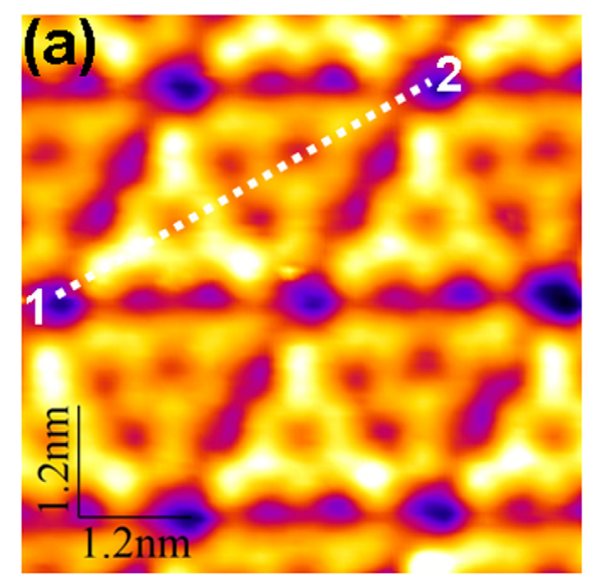

\section{(b)}
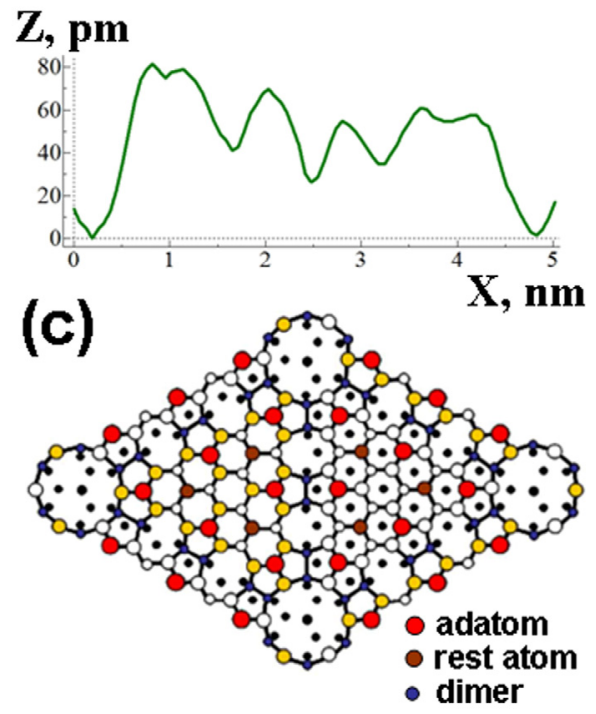

Fig. 2. (a) A raw occupied state $\mathrm{STM}$ image of the $\mathrm{Si}(111) 7 \times 7$ surface measured with a W[001] tip $\left(6 \times 6 \mathrm{~nm}^{2}, \mathrm{U}=-1.4 \mathrm{~V}, \mathrm{I}=50 \mathrm{~A}\right)$. (b) Cross-section $(1-2)$ of the image in panel (a). (c) Top view of the $\mathrm{Si}(111) 7 \times 7$ unit cell. 

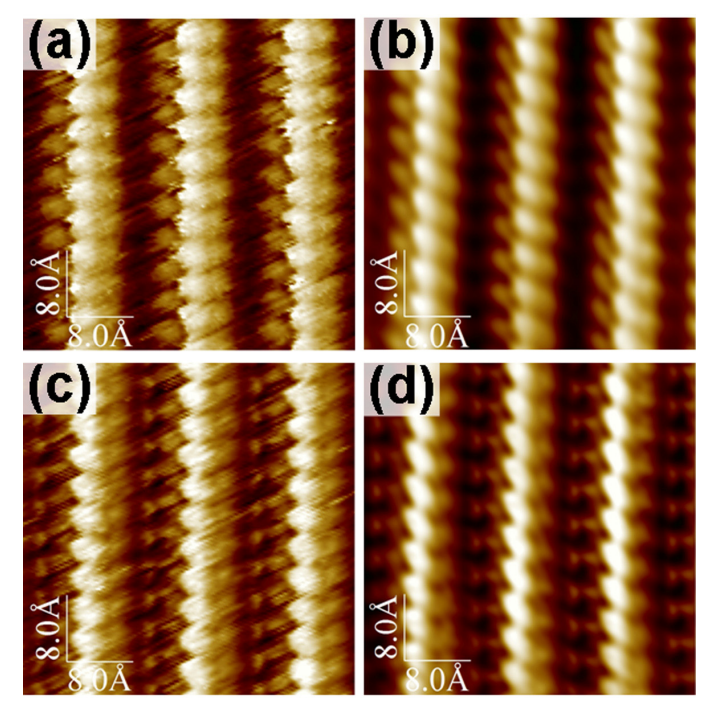

(e)

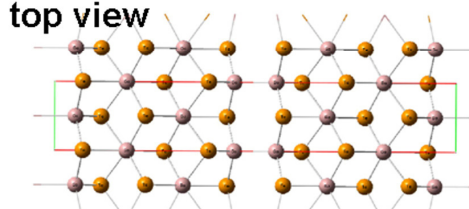

side view

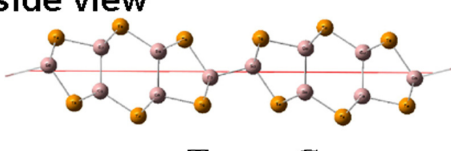

- Te - Ga

Fig. 3. (a-d) $4 \times 4 \mathrm{~nm}^{2} \mathrm{STM}$ images of the $\operatorname{GaTe}(10-2)$ surface obtained with a $\mathrm{W}$ [001] tip at $\mathrm{U}=-1.0 \mathrm{~V}, \mathrm{I}=120 \mathrm{pA}(\mathrm{a}, \mathrm{b})$ and $\mathrm{U}=-1.0 \mathrm{~V}, \mathrm{I}=50 \mathrm{pA}(\mathrm{c}, \mathrm{d})$. Images in panels $(b, d)$ were obtained from the raw data $(a, c)$ after removing thermal drift and smoothing by a $3 \times 3$ matrix using WSXM software [20]. (e) Schematic model of the GaTe( $10-2)$ reconstructed surface.

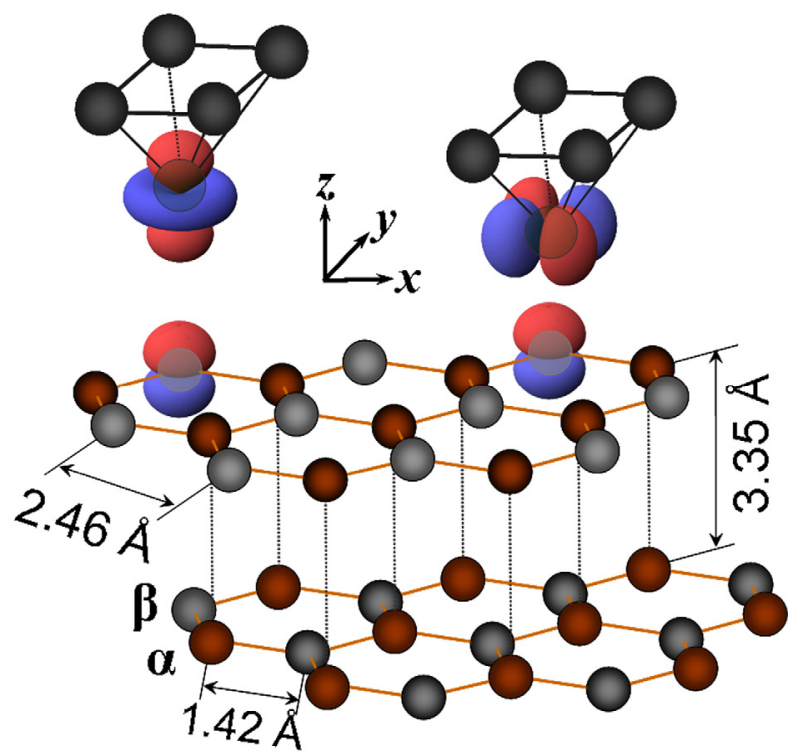

Fig. 4. Schematic view of a W[001] tip with the $d_{3 z-r}^{2}{ }^{2}$ (left) or $d_{x-y}^{2}{ }^{2}$ (right) orbital at the apex above the graphite surface. 

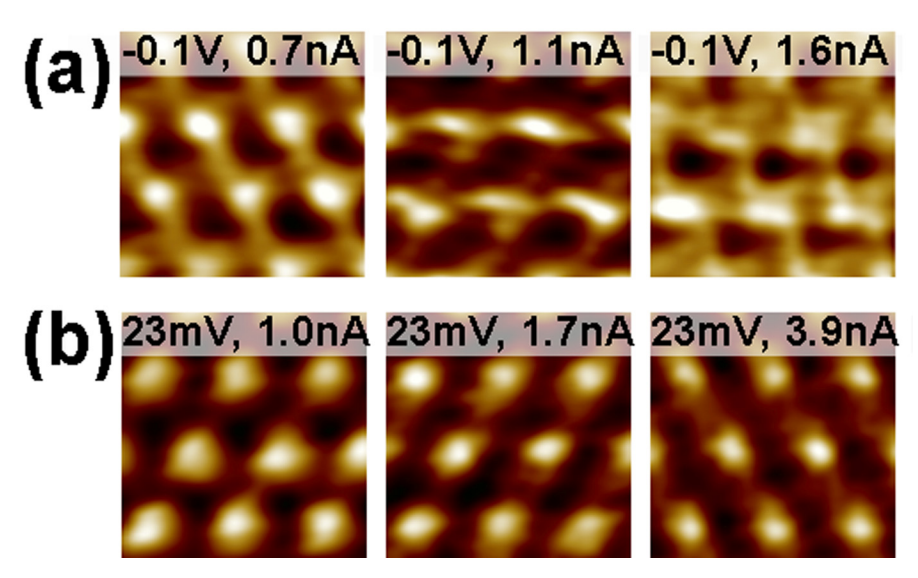

Fig. 5. Tunneling current dependences of HOPG(0001) STM images obtained with the W[001] tips with invariable structure throughout the scanning cycle at fixed bias voltages of $-0.1 \mathrm{~V}$ (a) and $23 \mathrm{mV}$ (b). The currents are indicated for each particular frame.
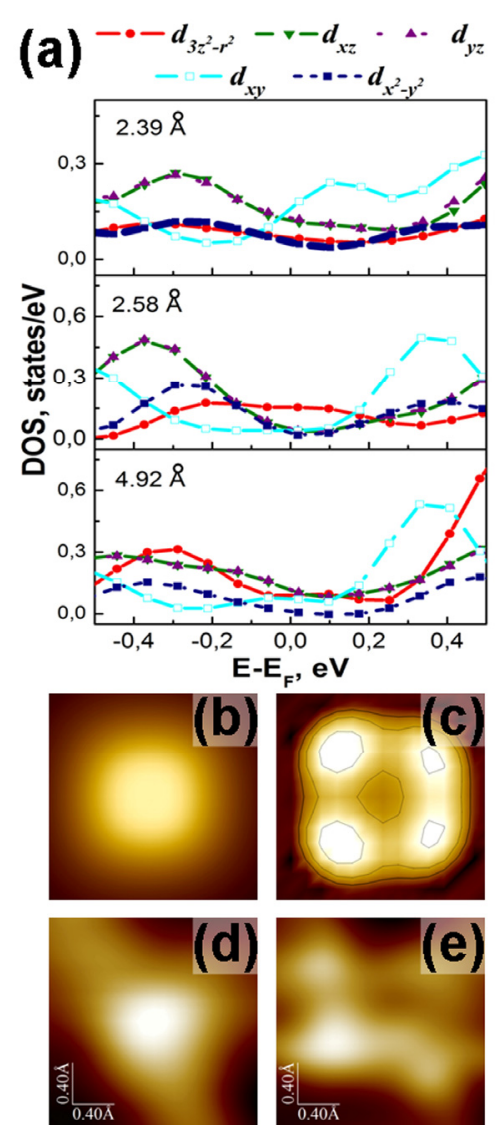

Fig. 6. (a) PDOS associated with different $d$-orbitals of the W[001] tip apex atom interacting with the HOPG(0001) surface. The final distances between tip and surface atom nuclei are indicated on each panel. $(b, c)$ Calculated charge density maps for W[001]-graphite systems in (001) planes located $0.8 \AA$ (b) and $0.45 \AA$ (c) above the apex atom at tip-sample distances of $2.71 \AA$ (b) and $2.39 \AA$ (c). (d,e) STM images of HOPG(0001) measured with W[001] tip at $\mathrm{U}=-0.1 \mathrm{~V}$ and $\mathrm{I}=0.7 \mathrm{nA}$ (d) and I=1.7 nA (e). 